\title{
Pharmacological manipulation of cannabinoid neurotransmission reduces neuroinflammation associated with normal aging
}

\author{
Isabelle Bardou ${ }^{1}$, Nicholas DiPatrizio ${ }^{2}$, Holly M. Brothers ${ }^{1}$, Roxanne M. Kaercher ${ }^{1}$, \\ Kevin Baranger ${ }^{3,4}$, Mollie Mitchem ${ }^{1}$, Sarah C. Hopp ${ }^{1}$, Gary L. Wenk ${ }^{1^{*}}$, Yannick Marchalant ${ }^{3,4}$ \\ ${ }^{1}$ Department of Psychology, Ohio State University, Columbus, USA; ${ }^{*}$ Corresponding Author: wenk.6@osu.edu \\ ${ }^{2}$ Department of Pharmacology, University of California, Irvine, USA \\ ${ }^{3}$ NICN, CNRS UMR 7259, Marseille, France \\ ${ }^{4}$ NICN, Aix-Marseille University, Marseille, France
}

Received 1 June 2012; revised 30 June 2012; accepted 10 July 2012

\begin{abstract}
We have previously demonstrated that antagonism of glutamate NMDA receptors or activation of endocannabinoid receptors could reduce experimentally induced neuroinflammation within the hippocampus of young rats. In the current study, we investigated whether pharmacological manipulation of glutamate or endocannabinoid neurotransmission could reduce naturally-occurring neuroinflammation within the hippocampus of aged rats. We investigated whether UCM707, an inhibitor of endocannabinoid re-uptake, WIN55,212-2, an endocannabinoid receptor agonist, and URB597, an inhibitor of endocannabinoid catabolism, or memantine, a non-competitive, low-affinity, inhibitor of the open NMDA receptor channel, could reduce the number of MHC II-IR microglia within the hippocampus. All of the drugs, except URB597, reduced the number of reactive microglia, as compared to vehicle treated rats. The current results suggest potential pharmacological approaches that may mitigate the pathological consequences of chronic brain inflammation associated with numerous neurodegenerative diseases.
\end{abstract}

Keywords: Rats; Memantine; UCM707; Microglia; WIN-55,212-2; URB597

\section{INTRODUCTION}

Neuroinflammation contributes to the pathogenesis of numerous age-related neurodegenerative disorders [1-3]. In the aged brain, naturally occurring neuroinflammation does not respond to anti-inflammatory drugs [4] which may explain the numerous failures of interventional studies using anti-inflammatory therapies in patients with Alzheimer's disease [5]. We therefore investigated some alternative approaches using drugs that we have previously shown could effectively reduce the pathological, neurochemical, molecular, genetic and behavioral expressions of experimentally-induced neuroinflammation in the young rat brain [6-12]. The current studied determined whether pharmacological manipulation of glutamate or endocannabinoid neurotransmission within the aged rat brain could reduce a critical indicator of neuroinflammation, the number of activated microglia.

The endocannabinoid system regulates aspects of the brain's inflammatory response, including the release of pro-inflammatory cytokines and modulation of microglial activation $[6-8,13,14]$. The endocannabinoid system includes two known ligands, anandamide (AEA) and 2-arachidonoyl-sn-glycerol (2-AG) that are produced and released "on demand" $[13,15,16]$. Anandamide binds to endocannabinoid and TRPV1 receptors [17] and is primarily inactivated by a member of the serine hydrolase family of enzymes known as fatty acid amide hydrolase (FAAH) [15]. URB597 is a potent, selective and ireversible inhibitor of FAAH [18] that is currently under investigation for its anti-depressant, anti-anxiety, neuronprotective and analgesic proclivities [19]. UCM707 is an endocannabinoid re-uptake inhibitor that can also attenuate the release of cytokines from LPS-exposed astrocytes and also provide neuroprotection from excitotoxic stimuli [20,21]. Memantine, an open-channel, non-competitive, low-affinity, N-methyl-d-aspartate (NMDA) recaptor inhibitor [22] is currently approved for the treatment of Alzheimer's disease. We have previously demonstrated that memantine can reduce the cognitive and pathological consequences of chronic neuroinflammation in young rats exposed to lipopolysaccharide $[10,12]$. 
The current study focused upon the response of naturally occurring, aged microglia within the hippocampus that express major histocompatibility complex class II (MHC II-IR). We focused upon the hippocampus because this gray matter region demonstrates the greatest concentration of MHC II-IR microglia with normal aging [7,11, 12].

\section{MATERIALS AND METHODS}

Eighty aged (24 months) male F-344 rats (Harlan Sprague-Dawley, Indianapolis, IN) were singly housed in Plexiglas cages with free access to food and water. The rats were maintained on a $12 / 12$-h light-dark cycle in a temperature-controlled room $\left(22^{\circ} \mathrm{C}\right)$ with lights off at 0800. All rats were given health checks, handled upon arrival and allowed at least one week to adapt to their new environment prior to surgery. We certify that the experiments were carried out in accordance with the National Institute of Health Guide for the Care and Use of Laboratory Animals (NIH Publications No. 80-23) revised 1996. We also certify that the formal approval to conduct the experiments has been obtained from the animal subjects review board from Ohio State University.

Rats were administered either memantine $(10 \mathrm{mg} / \mathrm{kg} /$ day, Tocris, Minneapolis, MN), WIN-55,212-2 (2.0 mg/kg/day, Tocris), UCM707 (5 mg/kg/day, Tocris), URB597 (Cayman Chemicals, Ann Harbor, MI, $0.3 \mathrm{mg} / \mathrm{kg} /$ day) or their vehicle (dimethylsulfoxide + polyethylene glycol 50/50 $\mathrm{v} / \mathrm{v}$, Sigma, St-Louis, MO). For histological analyses, each rat was deeply anesthetized with isoflurane and prepared for a transcardiac perfusion of the brain with cold saline containing $1 \mathrm{U} / \mathrm{ml}$ heparin, followed by $4 \%$ paraformaldehyde in $0.1 \mathrm{M}$ phosphate buffer, $\mathrm{pH}$ 7.4. The brains were then prepared as previously described $[7,8]$ using the monoclonal antibody OX-6 directed against MHC II antigen (final dilution 1, 200, BD Biosciences Pharmigen, San Diego, CA) to visualize MHC II-IR microglia cells. No staining was detected in the absence of the primary or secondary antibodies. Tissues were counterstained using cresyl violet. Quantification of cell density in the reconstructed hippocampal coronal sections was assessed using Nikon elements imaging software (Nikon Instruments, Melville, NY).

In order to confirm the effectiveness of the endocannabinoid catabolic inhibitor, a separate group of aged rats were injected (i.p.) with URB597 (0.3 or $1.0 \mathrm{mg} / \mathrm{kg}$ ) or its vehicle and then sacrificed two hours later in order to determine the efficacy of URB597 in the brain of the aged rats. UCM707 has no effect upon endogenous levels of endocannabinoids [20,21]. Bilateral hippocampi were isolated and snap-frozen in liquid $\mathrm{N}_{2}$, stored $\left(-80^{\circ} \mathrm{C}\right)$ and assayed for AEA, OEA, PEA and 2-AG. Frozen hippocampal tissues were homogenized in $1 \mathrm{ml}$ of methanol containing the following internal standards, [2H4]-OEA, [2H4]-PEA, and [2H4]-AEA (Nu-Chek Prep, Elysian, MN); [d8]-2-AG (Cayman Chemical). Lipids were extracted with chloroform $(2 \mathrm{vol})$ and washed with water (1 vol). Organic phases were collected and dried under a stream of $\mathrm{N}_{2}$. Fatty acid ethanolamides and 2-AG were fractionated by open-bed silica gel column chromatography, as previously described [23]. Eluted fractions were dried under $\mathrm{N}_{2}$ and reconstituted in $0.1 \mathrm{ml}$ of methanol for liquid chromatography/mass spectrometry. For AEA and 2-AG analyses, an 1100-LC system coupled to a 1946D-MS detector (Agilent Technologies, Inc., Palo Alto, CA) equipped with an electrospray ionization interface was used. Lipids were separated on a XDB Eclipse C18 column $(50 \times 4.6 \mathrm{~mm}$ i.d., $1.8 \mu \mathrm{m}$, Zorbax $)$, eluted by a gradient of methanol in water (from $85 \%$ to $90 \%$ methanol in $2.5 \mathrm{~min}$ ) at a flow rate of $1.5 \mathrm{ml} / \mathrm{min}$. Column temperature was kept at $40^{\circ} \mathrm{C}$. MS detection was in the positive ionization mode, capillary voltage was set at $3 \mathrm{kV}$, and fragmentor voltage at $120 \mathrm{~V}$. Lipids were quantified with an isotope-dilution method [24], monitoring sodium adducts of the molecular ions $[\mathrm{M}+\mathrm{Na}]^{+}$in the selected ion-monitoring mode.

All statistical analyses were performed using Statview software (SAS Institute Inc.). Results are expressed as means \pm SEM. Statistical analyses were performed using ANOVA.

\section{RESULTS}

Immunostaining (Figure 1) revealed MHC II-IR microglia (OX-6-immunopositive) cells distributed throughout the hippocampus. The MHC II-IR microglia had a characteristic bushy morphology with increased cell body size and contracted and ramified processes similar to that previously reported [6]. The number of MHC II-IR microglia per millimeter square was determined in three different areas of interest, as previously reported in detail [11], dentate gyrus (DG), CA3 and CA1 regions of the hippocampus (Figure 2). An ANOVA revealed an overall main effect of drug treatment in the $\mathrm{DG}\left(\mathrm{F}_{3,23}=20.514\right.$, $\mathrm{p}$ $<0.001)$, and CA3 $\left(\mathrm{F}_{3,23}=133.698, \mathrm{p}=0.028\right)$, but not in the CA1 region $\left(\mathrm{F}_{3,23}=4.677, \mathrm{p}=0.843\right)$. Memantine, WIN 55,212-2 and UCM-707 reduced significantly the number of immunostained microglia in the DG and the CA3 regions, as compared to vehicle treated rats $(\mathrm{p}<$ 0.001 and $p<0.05$, respectively). URB597 treatment, at either dose, produced no significant reduction in the number of MHC II-IR microglia, $\left(\mathrm{F}_{2,57}=0.212, \mathrm{p}=0.81\right)$. URB597 treatment produced a significant $(\mathrm{p}<0.05)$ increase in the endogenous levels of the endocannabinoids oleoylethanolamide (OEA), palmitoylethanolamide (PEA) or AEA without affecting 2-AG levels (Figure 3). 


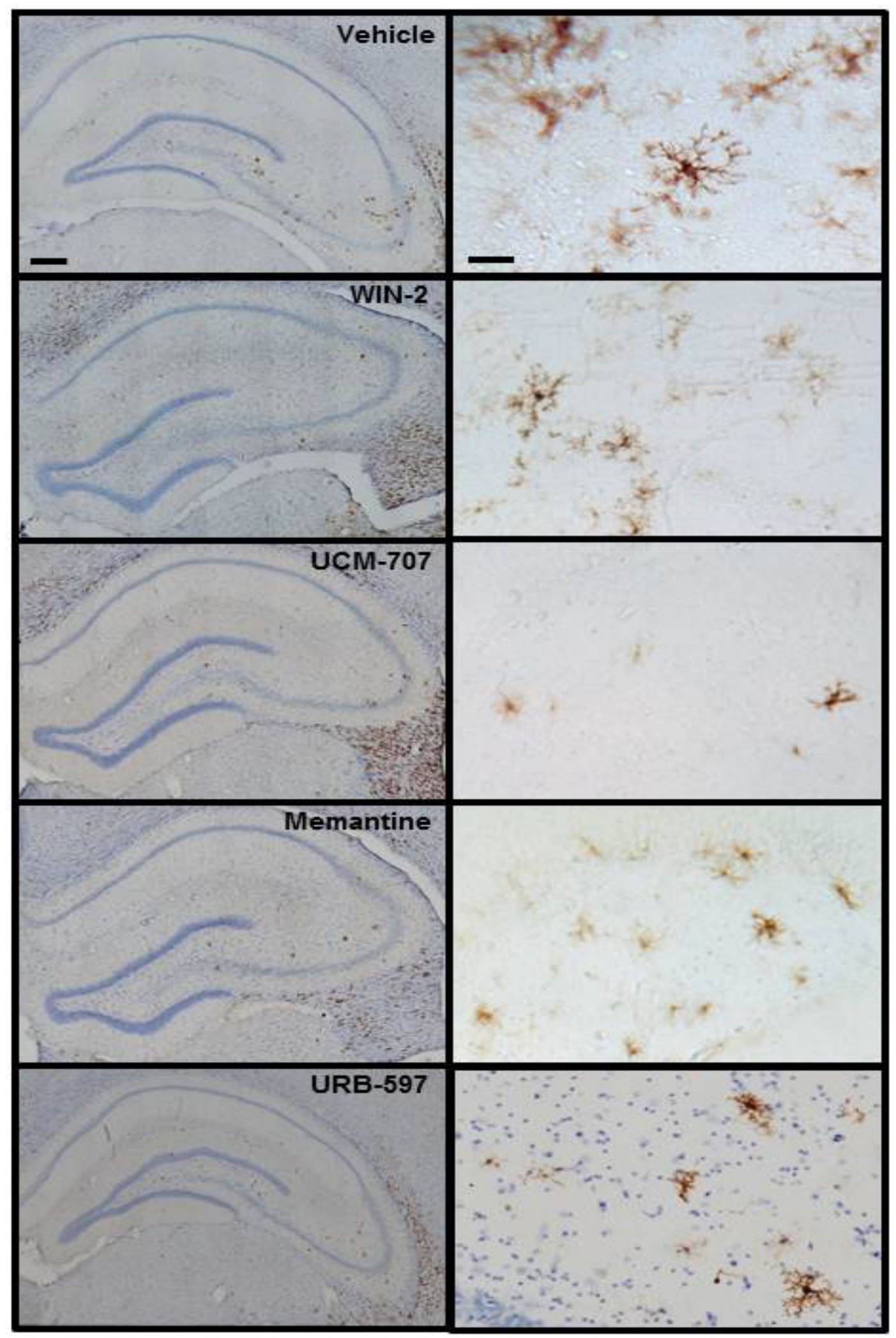

Figure 1. Coronal sections from the dorsal hippocampus of aged rats. MHC II-IR microglia (brown OX-6 immunoreactive cells) were particularly elevated within the granule cell layer and the hilar region of the dentate gyrus (DG) as well as inside and immediately around the CA3 molecular layer. No immunopositive staining was found in CA1. Nuclei are counterstained with cresyl violet. Scale bars: $100 \mu \mathrm{m}$ and $10 \mu \mathrm{m}$. 


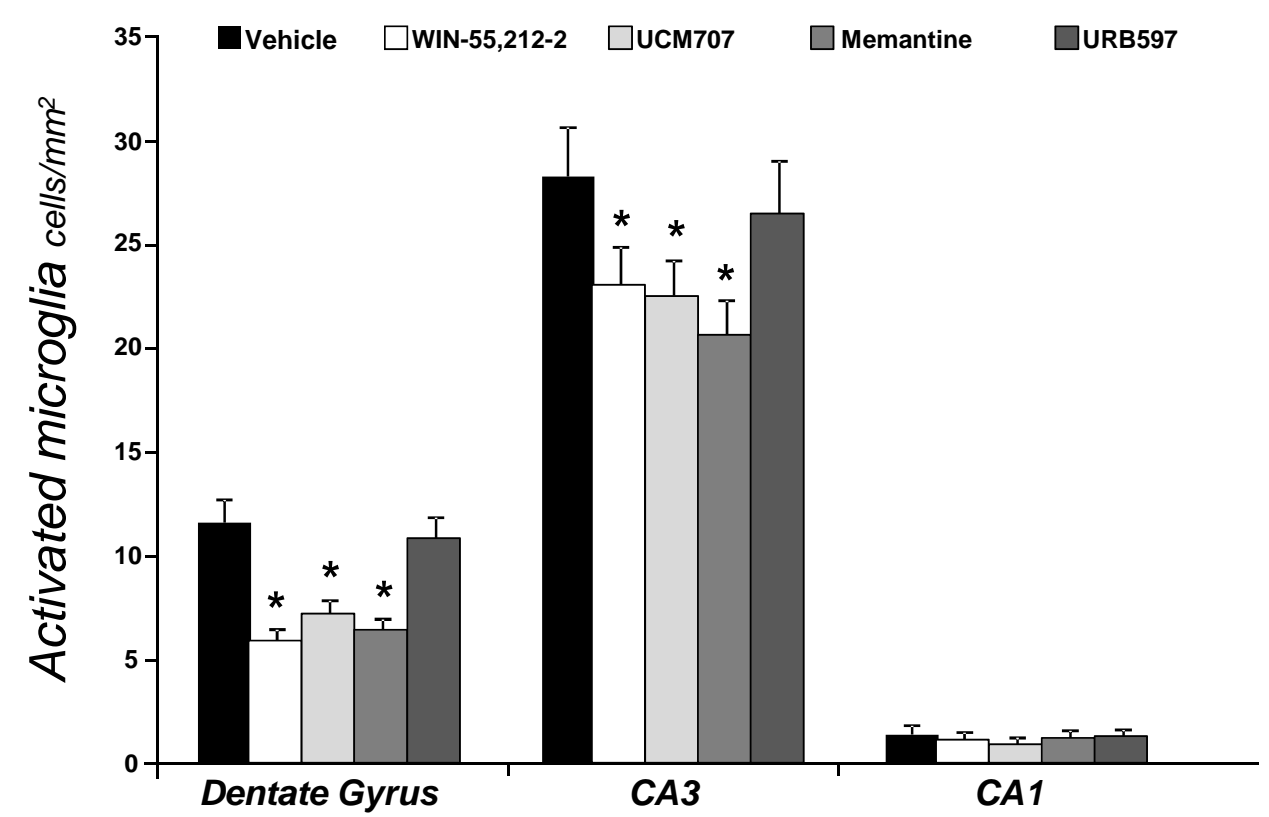

Figure 2. Density (number of cells per square millimeter) of OX-6 immunoreactive (MHC II-IR) microglia across three regions of interest in the hippocampus of aged rats following drug treatment. ${ }^{*}$ Indicates $\mathrm{p}<0.001$ for the DG, $\mathrm{p}<0.03$ for CA3 vs. the vehicle-treated group.
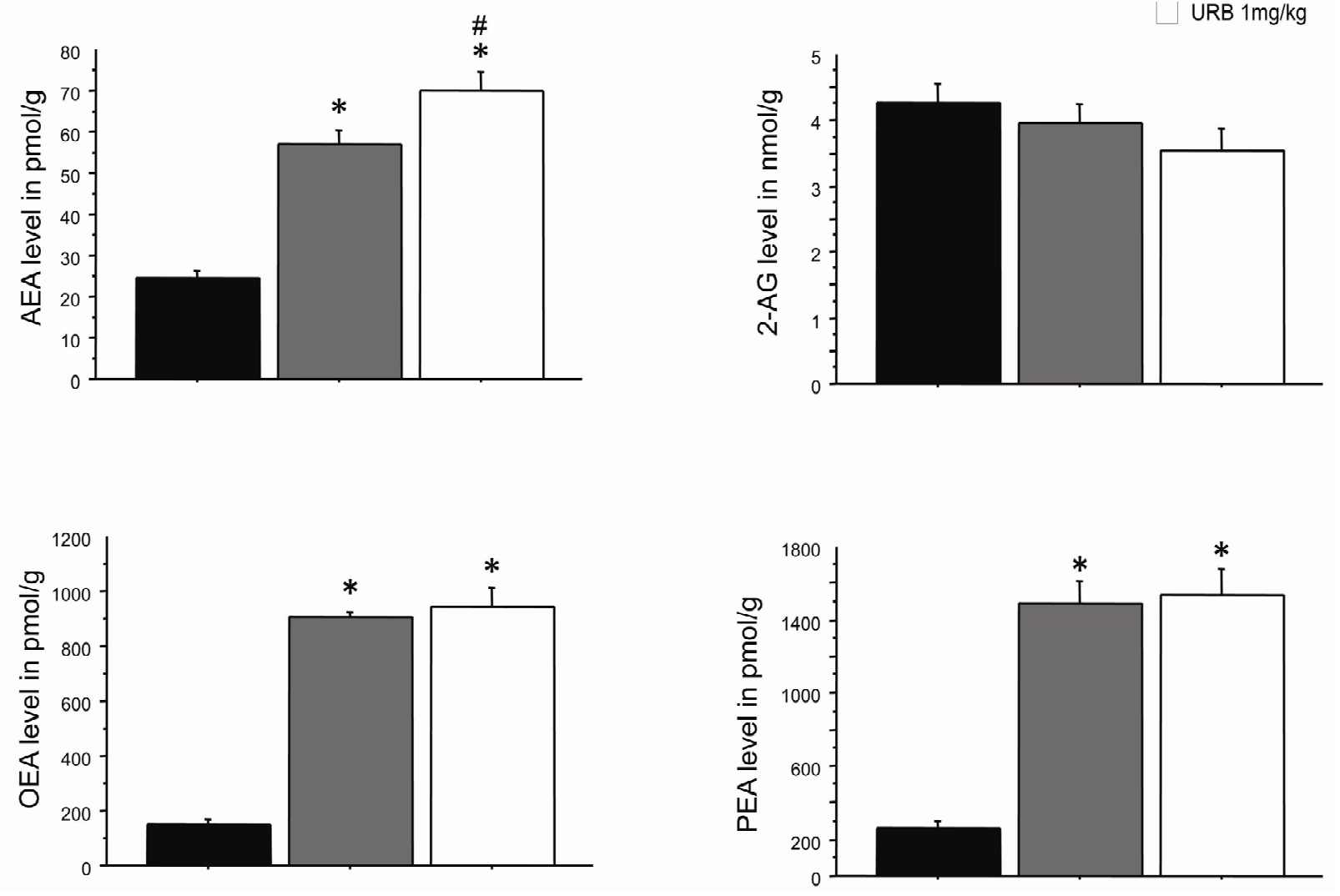

Figure 3. Endocannabinoids levels in the hippocampus following an i.p. injection of URB597 (0.3 or $1 \mathrm{mg} / \mathrm{kg})$. Tissues levels of AEA, OEA and PEA were significantly increased following administration of both doses. ${ }^{*} \mathrm{p}<0.05$ vs. control levels. ${ }^{\#}$ p $<0.05$ vs. $0.3 \mathrm{mg} / \mathrm{kg}$. No significant changes were observed in $2-A G$ levels. 


\section{DISCUSSION}

The current study demonstrated that pharmacological manipulation of glutamate or cannabinoid neurotransmission can significantly reduce the number of MHC II-IR microglia within the aged hippocampus. The results of the current study are consistent with our previous report that memantine can significantly reduce the number of MHC II-IR microglia in the hippocampus of young rats exposed to lipopolysaccharide; we now confirm its ability to reduce the number of naturally occurring MHC II-IR microglia within an aged brain [12]. The effects of memantine in the aged rat brain may result from a direct effect upon microglia, however, the lack of NMDA receptors on MHC II-IR microglia in the hippocampus [12] and the inability of memantine to alter the number of MHC II-IR microglia in culture following exposure to lipopolysaccharide [12], suggests that memantine reduced the number of microglia expressing MHC II by blocking NMDA receptors expressed on hippocampal neurons.

UCM707, a potent and selective endocannabinoid uptake inhibitor, reduced the number of MHC II-IR microglia in the hippocampus of aged rats. UCM707 has numerous proclivities that make it an attractive pharmaceutical invention in Alzheimer's disease; it can inhibit the production of pro-inflammatory mediators induced by lipopolysaccharide-stimulated astrocytes in vitro, as well as reduce the release of nitric oxide, and reduce the production of tumor necrosis factor-alpha and interleukin-1 beta [20] and it is neuroprotective effects against glutamate excitotoxicity [21].

Selective FAAH inhibition did not reduce the number of MHC II-IR microglia. The lowest dose of URB597 chosen for investigation $(0.3 \mathrm{mg} / \mathrm{kg})$ did significantly increase endogenous levels of the endocannabinoids AEA, OEA and PEA in young rats, consistent with previous reports [25-27]. The levels of endocannabinoids measured in the untreated aged rats our study are consistent with brain levels usually found in young rats [25-27], ruling out a potentially lower basal level of these molecules in an aged brain. Also, we have shown that the number of CB1 receptors neither increased nor decreased with normal aging $[9,28]$. When microglia are exposed to pro-inflammatory stimuli and become activated they rapidly up-regulate their expression of CB receptors [29]; the absence of $\mathrm{CB}$ receptor up-regulation suggests that the MHC II-IR microglia are not in a classical activated state in the aged hippocampus.

We have previously speculated that the ability of WIN-55,212-2 to reduce the expression of MHC II-IR by resident microglia was partially related to its antagonism at TRPV1 receptors $[9,28]$ that are expressed by microglia [30]. The lack of effects by AEA or PEA elevated by
FAAH inhibition in aged rats in the current study are consistent with our hypothesis regarding the antagonistic properties of WIN-55,212-2 on TRPV1 receptors; these results also confirm the therapeutic potential of blocking TRPV1 receptors in diseases associated with inflammatory processes.

Overall, the current results suggest novel pharmacological approaches directly and indirectly targeting the glutamatergic and endocannabinoid neural systems that may mitigate the pathological consequences of chronic brain inflammation associated with numerous neurodegenerative diseases more effectively than standard antiinflammatory therapies.

\section{ACKNOWLEDGEMENTS}

Supported by US Public Health Service, RO1 AG030331 and RO1 AG037320 to GLW

\section{REFERENCES}

[1] Akiyama, H., Barger, S., et al. (2010) Inflammation in Alzheimer's disease. Neurobiology of Aging, 21, 383-421. doi:10.1016/S0197-4580(00)00124-X

[2] Remarque, E.J., Bollen, E.L., Weverling-Rijnsburger, A.W., Laterveer, J.C., Blauw, G.J. and Westendorp, R.G.J. (2001) Patients with Alzheimer's disease display a proinflammatory phenotype. Experimental Gerontology, 36, 171-176. doi:10.1016/S0531-5565(00)00176-5

[3] Nelson, P.T., Somam, L.A. and Lavi, E. (2002) Microglia in diseases of the central nervous system. Annals of Medicine, 34, 491-500. doi:10.1080/078538902321117698

[4] Hauss-Wegrzyniak, B., Vraniak, P. and Wenk, G.L. (1999) The effects of a novel NSAID upon chronic neuroinflammation are age dependent. Neurobiology of Aging, 20, 305-313. doi:10.1016/S0197-4580(99)00028-7

[5] Imbimbo, B.P. (2009) An update on the efficacy of nonsteroidal anti-inflammatory drugs in Alzheimer's disease. Expert Opinion on Investigational Drugs, 18, 1147-1168. doi: $10.1517 / 13543780903066780$

[6] Marchalant, Y., Rosi, S. and Wenk, G.L. (2007) Antiinflammatory property of the cannabinoid agonist WIN55212-2 in a rodent model of chronic brain inflammation. Neuroscience, 144, 1516-1522. doi:10.1016/j.neuroscience.2006.11.016

[7] Marchalant, Y., Cerbai, F., Brothers, H.M. and Wenk, G.L. (2008) Cannabinoid receptor stimulation is anti-inflamematory and improves memory in old rats. Neurobiology of Aging, 29, 1894-1901.

doi:10.1016/j.neurobiolaging.2007.04.028

[8] Marchalant, Y., Brothers, H.M. and Wenk, G.L. (2008) Inflammation and aging, can endocannabinoids help? Biomedical Pharmacotherapy, 6, 212-217. doi:10.1016/j.biopha.2008.02.004

[9] Marchalant, Y., Brothers, H.M. and Wenk, G.L. (2009) Cannabinoid agonist WIN-55,212-2 partially restores 
neurogenesis in the aged rat brain. Molecular Psychiatry, 14, 1068-1071. doi:10.1038/mp.2009.62

[10] Willard, L.B., Hauss-Wegrzyniak, B., Danysz, W. and Wenk, G.L. (2000) The cytotoxicity of chronic neuroinflammation upon basal forebrain cholinergic neurons of rats can be attenuated by glutamatergic antagonism or cyclooxygenage-2 inhibition. Experimental Brain Research, 134, 58-65. doi:10.1007/s002210000446

[11] Rosi, S., Ramirez-Amaya, V., Vazdarjanova, A., Worley, P.F., Barnes, C.A. and Wenk, G.L. (2005) Neuroinflammation alters the hippocampal pattern of behaviorally induced arc expression. Journal of Neuroscience, 25, 723731. doi:10.1523/JNEUROSCI.4469-04.2005

[12] Rosi, S., Vazdarjanova, A., Ramirez-Amaya, V., Worley, P.F., Barnes, C.A. and Wenk, G.L. (2006) Memantine protects against LPS-induced neuroinflammation, restores behaviorally-induced gene expression and spatial learning in the rat. Neuroscience, 142, 1303-1315. doi:10.1016/j.neuroscience.2006.08.017

[13] Di Marzo, V., Melck, D., Bisogno, T. and De Petrocellis, L. (1998) Endocannabinoids, endogenous cannabinoid receptor ligands with neuromodulatory action. Trends in Neurosciences, 21, 521-528. doi:10.1016/S0166-2236(98)01283-1

[14] Cabral, G.A. and Griffin-Thomas, L. (2008) Cannabinoids as therapeutic agents for ablating neuroinflammatory disease. Endocrine Metabolism, Immune Disorders \& Drug Targets, 8, 159-172. doi: $10.2174 / 187153008785700118$

[15] Piomelli, D. (2003) The molecular logic of endocannabinoid signaling. Nature Reviews Neuroscience, 4, 873-884. doi: $10.1038 / \mathrm{nrn} 1247$

[16] Alger, B.E. and Kim, J. (2011) Supply and demand for endocannabinoids. Trends in Neurosciences, 34, 6-10. doi:10.1016/j.tins.2011.03.003

[17] Pertwee, R.G. (2005) Pharmacological actions of cannabinoids. Handbook of Experimental Pharmacology, 168, 1-51. doi:10.1007/3-540-26573-2_ 1

[18] Fegley, D., Gaetani, S., Duranti, A., Tontini, A., Mor, M. and Tarzia, G. (2005) Characterization of the fatty acid amide hydrolase inhibitor cyclohexyl carbamic acid 3'carbamoyl-biphenyl-3-yl ester (URB597), effects on anandamide and oleoylethanolamide deactivation. Journal of Pharmacology \& Experimental Therapeutics, 313, 352-358. doi:10.1124/jpet.104.078980

[19] Hwang, J., Adamson, C., Butler, D., Janero, D.R., Makriyannis, A. and Bahr, B.A. (2010) Enhancement of endocannabinoid signaling by fatty acid amide hydrolase inhibition, a neuroprotective therapeutic modality. Life
Sciences, 86, 615-623. doi:10.1016/j.lfs.2009.06.003

[20] Ortega-Gutierrez, S., Molina-Holgado, E. and Guaza, C. (2005) Effect of anandamide uptake inhibition in the production of nitric oxide and in the release of cytokines in astrocyte cultures. Glia, 52, 163-168. doi:10.1002/glia.20229

[21] Loría, F., Petrosino, S., et al. (2010) An endocannabinoid tone limits excitotoxicity in vitro and in a model of multiple sclerosis. Neurobiology of Disease, 37, 736-749.

[22] Rogawski, M. and Wenk, G.L. (2003) The neuropharmacological basis for Memantine in the treatment of Alzheimer's disease. CNS Drug Review, 9, 275-308. doi:10.1111/j.1527-3458.2003.tb00254.x

[23] Astarita, G. and Piomelli, D. (2009) Lipidomic analysis of endocannabinoid metabolism in biological samples. Journal of Chromatography B, 877, 2755-2767. doi:10.1016/i.jchromb.2009.01.008

[24] Giuffrida, A., Rodriguez de Fonseca, F., Nava, F. and Loubet-Lescoulie, P. (2000) Elevated circulating levels of anandamide after administration of the transport inhibitor, AM404. European Journal of Pharmacology, 408, 161168. doi:10.1016/S0014-2999(00)00786-X

[25] Eisenstein, S.A., Holmes, P.V. and Hohmann, A.G. (2009) Endocannabinoid modulation of amphetamine sensitization is disrupted in a rodent model of lesion-induced dopamine dysregulation. Synapse, 63, 941-950. doi:10.1002/syn.20679

[26] Haller, J., Barna, I., et al. (2009) Interactions between environmental aversiveness and the anxiolytic effects of enhanced cannabinoid signaling by FAAH inhibition in rats. Psychopharmacology, 204, 607-616. doi:10.1007/s00213-009-1494-7

[27] Ahn, K., Johnson, D.S., et al. (2007) Novel mechanistic class of fatty acid amide hydrolase inhibitors with remarkable selectivity. Biochemistry, 46, 13019-13030. doi:10.1021/bi701378g

[28] Marchalant, Y., Brothers, H.M., Norman, G.J., Karolina, K., De Vries, C. and Wenk, G.L. (2009) Cannabinoids attenuate the effects of aging upon neuroinflammation and neurogenesis. Neurobiology of Disease, 34, 300-307. doi:10.1016/i.nbd.2009.01.014

[29] Stella, N. (2009) Endocannabinoid signaling in microglia cells. Neuropharmacology, 56, 244-253. doi:10.1016/j.neuropharm.2008.07.037

[30] Jiang, X., Newell, E.W. and Schlichter, L.C. (2003) Regulation of TRPM7-like current in rat brain microglia. Journal of Biological Chemistry, 278, 42867-42876. doi:10.1074/jbc.M304487200 